\title{
Acute pulmonary embolism: mortality prediction by the 2014 European Society of Cardiology risk stratification model
}

\author{
Cecilia Becattini ${ }^{1}$, Giancarlo Agnelli ${ }^{1}$, Mareike Lankeit ${ }^{2}$, Luca Masotti ${ }^{3}$, \\ Piotr Pruszczyk ${ }^{4}$, Franco Casazza ${ }^{5}$, Simone Vanni ${ }^{6}$, Cinzia Nitti ${ }^{7}$, \\ Pieter Kamphuisen ${ }^{8}$, Maria Cristina Vedovati ${ }^{1}$, Maria Grazia De Natale ${ }^{1}$ and \\ Stavros Konstantinides ${ }^{2}$
}

\begin{abstract}
Affiliations: ${ }^{1}$ Internal and Cardiovascular Medicine-Stroke Unit, University of Perugia, Perugia, Italy. ${ }^{2}$ Center for Thrombosis and Hemostasis, University Medical Center Mainz, Mainz, Germany. ${ }^{3}$ Internal Medicine, Santa Maria Nuova Hospital, Florence, Italy. ${ }^{4}$ Dept of Internal Medicine and Cardiology, Warsaw Medical University, Warsaw, Poland. ${ }^{5}$ Ospedale San Carlo Borromeo, Milano, Italy. ${ }^{6}$ Azienda Ospedaliero-Universitaria Careggi, Florence, Italy. ${ }^{7}$ Azienda Ospedaliera-Universitaria, Ancona, Italy. ${ }^{8}$ Dept of Vascular Medicine, University Medical Center Groningen, Groningen, The Netherlands.
\end{abstract}

Correspondence: Cecilia Becattini, Internal and Cardiovascular Medicine-Stroke Unit, University of Perugia, Perugia 06132, Italy. E-mail: cecilia.becattinidunipg.it

ABSTRACT The European Society of Cardiology (ESC) has proposed an updated risk stratification model for death in patients with acute pulmonary embolism based on clinical scores (Pulmonary Embolism Severity Index (PESI) or simplified PESI (sPESI)), right ventricle dysfunction (RVD) and elevated serum troponin (2014 ESC model).

We assessed the ability of the 2014 ESC model to predict 30-day death after acute pulmonary embolism. Consecutive patients with symptomatic, confirmed pulmonary embolism included in prospective cohorts were merged in a collaborative database. Patients' risk was classified as high (shock or hypotension), intermediate-high (RVD and elevated troponin), intermediate-low (RVD or increased troponin or none) and low (sPESI 0). Study outcomes were death and pulmonary embolism-related death at 30 days.

Among 906 patients (mean \pm SD age 68 $\pm 16,489$ females), death and pulmonary embolism-related death occurred in $7.2 \%$ and $4.1 \%$, respectively. Death rate was $22 \%$ in "high-risk" (95\% CI 14.0-29.8), $7.7 \%$ in "intermediate-high-risk" (95\% CI 4.5-10.9) and 6.0\% in "intermediate-low-risk" patients (95\% CI 3.4-8.6). One of the 196 "low-risk" patients died (0.5\%, 95\% CI 0-1.0; negative predictive value 99.5\%).

By using the 2014 ESC model, RVD or troponin tests would be avoided in about $20 \%$ of patients (sPESI 0 ), preserving a high negative predictive value. Risk stratification in patients at intermediate risk requires further improvement.

@ERSpublications

2014 ESC model in PE accurately identifies low-risk patients; further improvement for intermediate risk is needed http://ow.ly/10b3Qg 


\section{Introduction}

Acute pulmonary embolism is a common, potentially life-threatening disease [1]. In patients with acute pulmonary embolism, the risk of death is particularly high in the first days and then declines over time [2]. Therefore, risk stratification for short-term death is recommended to drive clinical management in the acute phase [3]. Clinical presentation and related scores, right ventricle dysfunction (RVD) and increased levels of serum troponin were shown to be associated with the risk of early death [4-8].

A risk stratification model was proposed in the 2008 European Society of Cardiology (ESC) guidelines on the clinical management of patients with acute pulmonary embolism [9]. This model was updated in the 2014 ESC guidelines by introducing two major changes in the risk stratification of haemodynamically stable patients [3]. First, the Pulmonary Embolism Severity Index (PESI) or simplified PESI (sPESI) drive further prognostic assessment. Indeed, PESI or sPESI identify patients at "low risk", i.e. those in which proceeding with risk stratification is not considered necessary, and patients at "intermediate risk", i.e. those that should proceed to the evaluation of RVD and serum troponin. Second, among intermediate-risk patients, those with both RVD and elevated troponin are categorised as at "intermediate-high" risk while all the other patients (those with either RVD or elevated troponin and with both absent) are categorised as at "intermediate-low" risk.

The aim of this study is to assess the ability of the 2014 ESC model to predict 30-day mortality in a population of patients with acute pulmonary embolism obtained by merging disease-specific prospective databases from four European countries. We also compared the prognostic value of the 2014 and 2008 ESC models.

\section{Methods}

\section{Study design and patient population}

Consecutive patients with symptomatic, objectively confirmed pulmonary embolism from 2010 up to 2014 were included in prospective cohorts (three cohorts) that were subsequently merged in a collaborative database. Patients were included in the study provided that data on the sPESI score, RVD and serum troponin as well as data on 30-day mortality were available.

sPESI score was assessed as previously described ( 1 point for each of the following: age $>80$ years, systolic blood $<100 \mathrm{mmHg}$, heart rate $\geqslant 110$ beats $\mathrm{min}^{-1}$, oxygen saturation $<90 \%$, history of cancer, congestive heart failure or pulmonary diseases) [5]. RVD was assessed by either echocardiography or computed tomography (CT) angiography. At echocardiography, RVD was defined by the presence of at least one of the following: 1) right-to-left ventricle end-diastolic diameter ratio $>1$ in apical four-chamber view, 2) right-to-left ventricle end-diastolic diameter ratio $>0.6$ in parasternal long-axis or subcostal four-chamber view and 3) right ventricle/right atrial pressure gradient $>30 \mathrm{mmHg}$ [10]. RVD was not considered of acute onset in the presence of right ventricle wall thickness $>7 \mathrm{~mm}$ or documentation of right ventricle overload at previous examinations. At CT angiography, RVD was defined as a right-to-left maximum dimension ratio $>0.9$ when measured in the two-dimension axial transverse images at the valvular plane [10]. Serum troponin levels were evaluated as normal or elevated by the local assays and according to local cut-off values.

The study was approved by the Institutional Review Board of the coordinating centre.

Study outcomes were death and pulmonary embolism-related death at 30 days. The cause of in-hospital death was adjudicated by the local investigator at each participating centre. Pulmonary embolism was considered the cause of death based on the autopsy or objective testing performed before death or in case of sudden death that could not be explained by a more compelling alternative diagnosis [11].

\section{Study analysis}

Frequency data are presented as proportions with 95\% confidence intervals. Continuous data are shown as mean \pm SD. Students t-test and the Chi-squared test or Fisher's exact test were used for comparisons of continuous and nominal variables, respectively. Logistic regression analysis was used to calculate the risk of death and death or clinical deterioration in different risk categories according to the ESC model. For the purpose of this study, haemodynamic status, sPESI score, RVD and increased troponin were evaluated as predictors of study outcomes.

Class-specific risks for death and death or clinical deterioration were calculated using logistic regression analysis. The ability of the 2014 ESC model to correctly classify haemodynamically stable subjects into one risk category in comparison with the 2008 ESC model was assessed by the $c$-statistic and the discrimination slope [12].

\section{Results}

1071 patients with acute pulmonary embolism were considered for inclusion in this study and 906 were ultimately included. Reasons for noninclusion are reported in figure 1. Demographic features and risk 


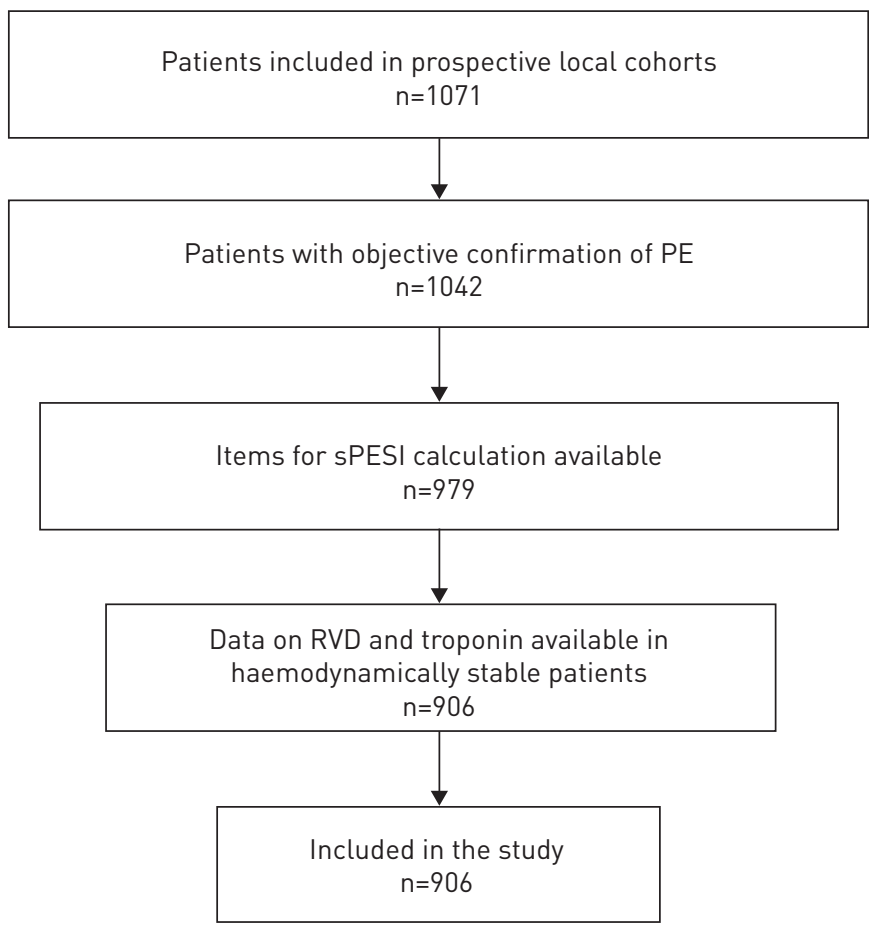

FIGURE 1 Study flow diagram. PE: pulmonary embolism; sPESI: simplified Pulmonary Embolism Severity Index; RVD: right ventricle dysfunction.

factors for death in the study population are reported in table 1. At presentation, 105 patients (11.7\%) were haemodynamically unstable and 801 haemodynamically stable. All patients received anticoagulant treatment and $11 \%$ also received systemic thrombolysis.

\section{Clinical course at 30 days}

Death at 30 days occurred in 65 patients $(7.2 \%, 95 \%$ CI $5.5-8.8 \%)$ and was deemed to be due to pulmonary embolism in 37 patients $(4.1 \%, 95 \%$ CI $2.8-5.4 \%$ ). Rates of death were $22.6 \%$ and $5.1 \%$ (odds ratio (OR) $1.43,95 \% \mathrm{CI} 1.19-1.73, \mathrm{p}<0.001)$ and rates of death due to pulmonary embolism were $16.0 \%$ and $2.5 \%$ (OR $1.66,95 \%$ CI $1.23-2.24, \mathrm{p}<0.001)$ in haemodynamically unstable and haemodynamically stable patients, respectively.

Clinical outcome in haemodynamically stable patients according to risk predictors

Among haemodynamically stable patients (801 patients), sPESI 0 was found in 196 patients (24.5\%) and sPESI $\geqslant 1$ in 605 patients (76.5\%). RVD was found in 462 patients (57.8\%). Increased troponin levels were

$\begin{array}{lc}\text { TABLE } 1 \text { Demographic features and risk factors for death } & \\ \text { Subjects } & 906 \\ \text { Age (range) years } & 68 \pm 16(18-98) \\ \text { Male } & 417(46) \\ \text { Shock or sustained hypotension } & 105(11.6) \\ \text { sPESI } & \\ 0 & 196(22) \\ 1 & 388(43) \\ 2 & 191(21) \\ 3 & 95(10) \\ 4 & 34(4) \\ 5 & 2 \\ \text { RVD } & \\ \text { At computed tomography } & 291 / 445(65) \\ \text { At echocardiography } & 436 / 855(51) \\ \text { Increased troponin } & 487 / 905(54)\end{array}$

Data are presented as $\mathrm{n}$, mean \pm SD (range), $\mathrm{n}(\%)$ or $\mathrm{n} / \mathrm{N}(\%)$. sPESI: simplified Pulmonary Embolism Severity Index; RVD: right ventricle dysfunction. 
found in 422 patients (52.8\%). The distribution of risk factors for death across different risk categories according to the 2014 ESC model is reported in table 2. Death at 30 days occurred in one patient $(0.5 \%)$ among those with sPESI 0 and in $41(5.2 \%)$ haemodynamically stable patients with sPESI $\geqslant 1(6.8 \%)$. Death due to pulmonary embolism occurred in one patients with sPESI $0(0.5 \%)$ and in 20 haemodynamically stable patients with sPESI $\geqslant 1(3.3 \%)$.

Death at 30 days occurred in 30 patients (6.5\%) with RVD and in 11 patients $(3.3 \%)$ without RVD (OR 2.06, 95\% CI 1.02-4.18, $\mathrm{p}=0.03)$; death due to pulmonary embolism was observed in 18 patients $(3.9 \%)$ with RVD and in two patients (0.6\%) without RVD (OR 6.81, 95\% CI 1.57-29.55, p=0.003).

Death at 30 days occurred in 29 patients (6.9\%) with increased serum troponin and in 12 patients $(3.2 \%)$ with normal serum troponin (OR 2.25, 95\% CI 1.13-4.48, $\mathrm{p}=0.02$ ); death due to pulmonary embolism was observed in 14 patients (3.3\%) with increased serum troponin and in six patients (1.3\%) with normal troponin (OR 2.13, 95\% CI 0.81-5.59, $\mathrm{p}=0.11$ ).

Death according to the 2014 and 2008 ESC risk stratification models

The 2014 ESC model classified 105 patients (11.6\%) as "high risk", 272 (30.0\%) as "intermediate-high risk", 333 as "intermediate-low risk" (36.7\%) and 196 as "low-risk" (21.6\%).

Death at 30 days occurred in $22 \%$ of "high-risk" patients (95\% CI 14.0-29.8), $7.7 \%$ of "intermediatehigh-risk" patients (95\% CI 4.5-10.9), 6.0\% of "intermediate-low-risk" patients (95\% CI 3.4-8.6) and 0.5\% of "low-risk" patients (95\% CI 0-1.0) (table 3). Among patients in the "intermediate-low" risk category, death at 30 days occurred in $8.9 \%$ (18 out of 203) and 1.5\% (two out of 130) of the patients with either RVD or increased serum troponin as compared with patients with none of these findings. One "low-risk" patient died due to pulmonary embolism; in this patient RVD was shown by both echocardiography and CT angiography. The negative predictive values for death at 30 days of "low-risk" patients (sPESI 0) and "intermediatelow-risk" patients were $99.5 \%$ and $94.0 \%$, respectively. The positive predictive value for "intermediatehigh-risk" patients was $7.7 \%$. The time course of death at 30 days according to 2014 ESC model risk categories is reported in figure $2 \mathrm{a}$.

Death due to pulmonary embolism at 30 days occurred in $15.2 \%$ of "high-risk" patients (95\% CI 8.4-22.1), $4.8 \%$ of "intermediate-high-risk" patients (95\% CI 2.2-7.3), 2.1\% of "intermediate-low-risk" patients $(95 \%$ CI $0.6-3.6)$ and $0.5 \%$ of "low-risk" patients (95\% CI 0-1.5). The time course of death due to pulmonary embolism according to 2014 ESC model risk categories is reported in figure $2 \mathrm{~b}$.

The 2008 ESC model classified 105 patients as "high risk" (11.6\%), 578 as "intermediate risk" (63.8\%) and 223 as "low risk" (27.8\%). The rates of death at 30 days were $21.9 \%, 6.9 \%$ and $0.9 \%$ in the three risk classes, respectively. Death due to pulmonary embolism occurred in $16.7 \%, 3.6 \%$ and $0 \%$ of patients, respectively.

The 2014 and 2008 ESC models showed similar discriminatory powers for death at 30 days (c-statistic $0.71,95 \%$ CI $0.65-0.77$ versus $0.71,95 \%$ CI $0.64-0.77$ ) as well as for pulmonary embolism-related death (c-statistic $0.75,95 \%$ CI $0.68-0.83$ versus 0.76 , 95\% CI $0.69-0.83$ ) (table 4 and figure 3 ). Similar results were obtained in terms of discriminatory power after adjusting for the use of thrombolytic treatment.

TABLE 2 Distribution of risk factors across different European Society of Cardiology (ESC) risk categories

\begin{tabular}{|c|c|c|c|c|c|}
\hline & All patients & \multicolumn{4}{|c|}{ ESC 2014 risk category } \\
\hline Subjects & 906 & 196 & 333 & 272 & 105 \\
\hline Male & $417(46)$ & $109(56)$ & $150(45)$ & $108(40)$ & $50(48)$ \\
\hline RVD & $535 / 906$ (59) & $81 / 196(41)$ & $110 / 333$ (33) & $272 / 272(100)$ & $72 / 105(69)$ \\
\hline Increased troponin & 487/905 (54) & $59 / 196(30)$ & $92 / 333$ (28) & $272 / 272(100)$ & $64 / 105(61)$ \\
\hline COPD & $108 / 906(12)$ & & $54 / 333(16)$ & $38 / 272(14)$ & $16 / 105$ (15) \\
\hline Recent surgery & $78 / 829$ (9) & $11 / 186(6)$ & $28 / 308(7)$ & $25 / 237(10)$ & $14 / 98(14)$ \\
\hline Recent trauma & $46 / 730(6)$ & $12 / 159(7)$ & $15 / 264(6)$ & $17 / 217(8)$ & $2 / 90(2)$ \\
\hline
\end{tabular}

Data are presented as $\mathrm{n}$, mean \pm SD (range), $\mathrm{n}(\%)$ or $\mathrm{n} / \mathrm{N}(\%)$. RVD: right ventricle dysfunction; COPD: chronic obstructive pulmonary disease. 
TABLE 3 Study outcome events in patients in different risk categories according to 2014 and 2008 European Society of Cardiology (ESC) models

Risk

ESC 2014

SPESI RVD or increased
troponin

Death at
30 days

$\geqslant 1$

High $^{\#}$

Intermediate-high $\geqslant 1$

Intermediate-low $\geqslant 1$

Low
0
23/105

(22, 14.0-29.8)

$21 / 272$

$(7.7,4.5-10.9)$

$20 / 333$

Either present

or both absent
$(6.0,3.4-8.6)$

$1 / 196$

$(0.5,0-1.5)$
ESC 2008

$\begin{gathered}\text { Death due to } \\ \text { pulmonary } \\ \text { embolism }\end{gathered}$
$16 / 105$
$(15.2,8.4-22.1)$
$13 / 272$
$(4.8,2.2-7.3)$
$7 / 333$
$(2.1,0.6-3.6)$
$1 / 196$
$(0.5,0-1.5)$

RVD or increased
troponin

Death at

30 days

$23 / 105$

(22, 14.0-29.8)

$21 / 307$

$(6.8,4.0-9.7)$

$19 / 271$

Either present

Both absent
(7.0, 4.0-10.0)

$2 / 223$

$(0.9,0-2.1)$

\section{Death due to}

pulmonary

embolism

$16 / 105$

(15.2, 8.4-22.1)

$13 / 307$

$(4.2,2.0-6.5)$

$8 / 271$

$(3.0,0.9-5.0)$

$0 / 223$

Data are presented as n/N (\%, 95\% Cl). RVD: right ventricle dysfunction. " : shock or sustained hypotension.

\section{Discussion}

This study shows that the 2014 ESC model efficiently stratifies patients with acute pulmonary embolism in categories at different risk of 30-day death and death due to pulmonary embolism.

Risk stratification for early death is a crucial step for the clinical management of acute pulmonary embolism. In this view, patients with high risk should proceed to rapid revascularisation either by pharmacologic or percutaneous catheter-directed or surgical treatment; patients with intermediate risk should receive antithrombotic therapy and close clinical surveillance to upgrade treatment in case of clinical deterioration [13]; patients with low risk can be candidates to home anticoagulant treatment or short hospital stay. Home treatment or short hospital stay in low-risk patients could be made feasible and easier with the use of new oral anticoagulant agents. The clinical relevance of risk stratification in patients with acute pulmonary embolism has been emphasised by the evolving scenario of treatment options.

The 2008 ESC model categorised a higher proportion of patients as "low risk" compared with the 2014 ESC model. However, the use of the 2008 model requires laboratory and instrumental examination in all patients. By introducing the sPESI score to drive further assessments, the 2014 ESC model avoids additional procedures in about $20 \%$ of patients nonetheless preserving a high negative predictive value and a similar $c$-statistic.

In our study, $41 \%$ of the patients categorised as "low risk" according to the sPESI score had RVD at CT angiography or at echocardiography and $30 \%$ had increased levels of serum troponin. One of these "low-risk" patients with RVD died due to pulmonary embolism (1.2\%). This patient had evidence of RVD at both echocardiography and CT angiography. According to the 2014 ESC model, RVD assessment is not necessary in patients at "low risk" (SPESI 0). However, the ease of obtaining information on RVD in patients who have CT angiography for the diagnosis of pulmonary embolism should support physicians to
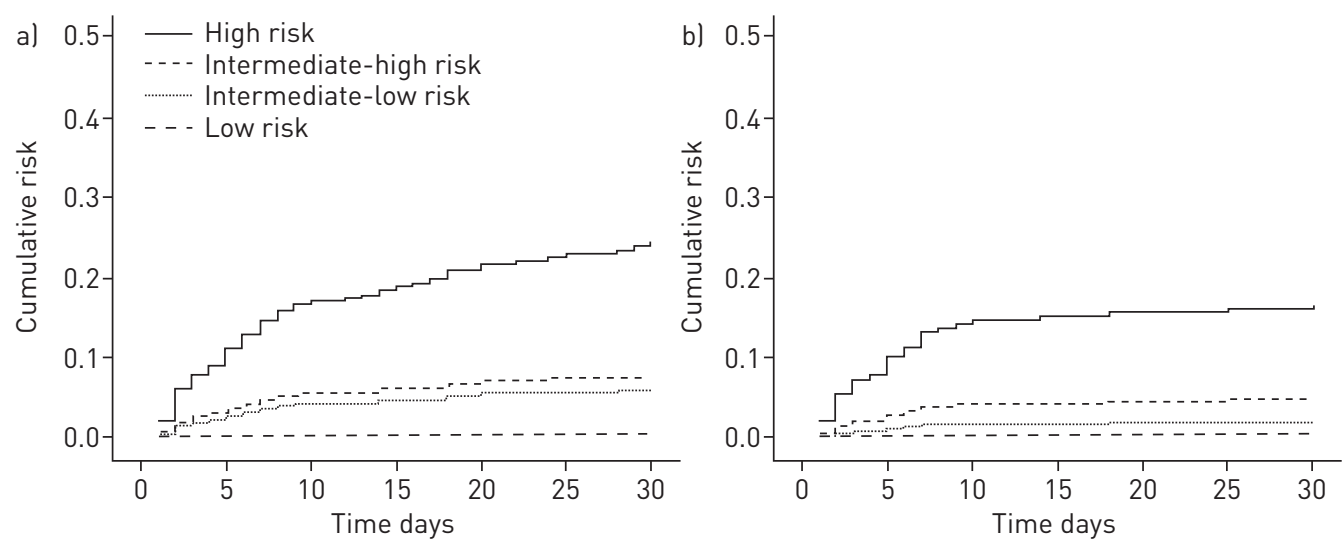

FIGURE 2 Risk of a) death at 30 days and b) death due to pulmonary embolism in patients belonging to different risk categories according to the 2014 European Society of Cardiology model. 
TABLE 4 Discriminatory power (c-statistic) for death at 30 days and for death due to pulmonary embolism for the 2014 and 2008 European Society of Cardiology (ESC) models

Death at 30 days

$0.71(0.65-0.77)$

2014 ESC model

2008 ESC model
Death due to pulmonary embolism

$0.75(0.68-0.83)$

$0.76(0.69-0.83)$

Data are presented as $\mathrm{n}(95 \% \mathrm{Cl})$.

claim for RVD assessment in every patient, particularly those that are candidates for home treatment. Whether the fatal case observed among patients with sPESI 0 should induce to consider the need for further risk stratification in these patients requires further evaluation in large prospective studies. The relatively low $1.2 \%$ mortality rate observed in "low-risk" patients with RVD suggests that the predictive value of RVD should be considered along with the clinical risk assessment.

The difference in the risk of death in patients at "intermediate-high" and "intermediate-low" risk according to the 2014 ESC model is relatively modest. Whether such a difference is high enough to justify treatment upgrading in intermediate-high-risk patients remains a matter of debate. This seems to be particularly relevant as treatment upgrading is associated with an increased risk of adverse event, as it is the case for bleeding associated to thrombolysis.

In intermediate-risk patients, the spectrum of clinical outcomes is particularly wide and probably influenced by risk factors other than those explored by the assessment of RVD or injury. We found a higher prevalence of cancer in patients at intermediate-low compared to intermediate-high risk. Whether this difference or other potential unexplored differences played a role in influencing mortality remains undefined. Further studies are required to improve the risk profile of patients at intermediate risk. Whether this improvement will derive from a better definition of the cut-off value of RVD or injury or from new approaches is difficult to anticipate.

According to the 2014 ESC model, the difference in the risk of death due to pulmonary embolism in patients at intermediate-high and intermediate-low risk was more pronounced. Indeed, risk stratification in the 2014 ESC model is based on disease-specific predictors of death. Strategies integrating haemodynamic variables or plasma lactate levels with RVD and troponin seem able to identify normotensive patients at particularly high risk of death [14-16]. Further evidence is needed to assess the clinical value of these observations.

In our study, the rates of high-risk patients (11.6\%) and low-risk patients according to sPESI (21.6\%) suggest the inclusion of a population at higher risk of death than in previous studies in patients with acute pulmonary embolism. This could be due to the higher prevalence of cardiology units over emergency departments among study centres in our study compared with previous studies. Whether this peculiar composition of the study population might explain the modest discrimination ability of the ESC model for the intermediate-risk category remains to be defined.
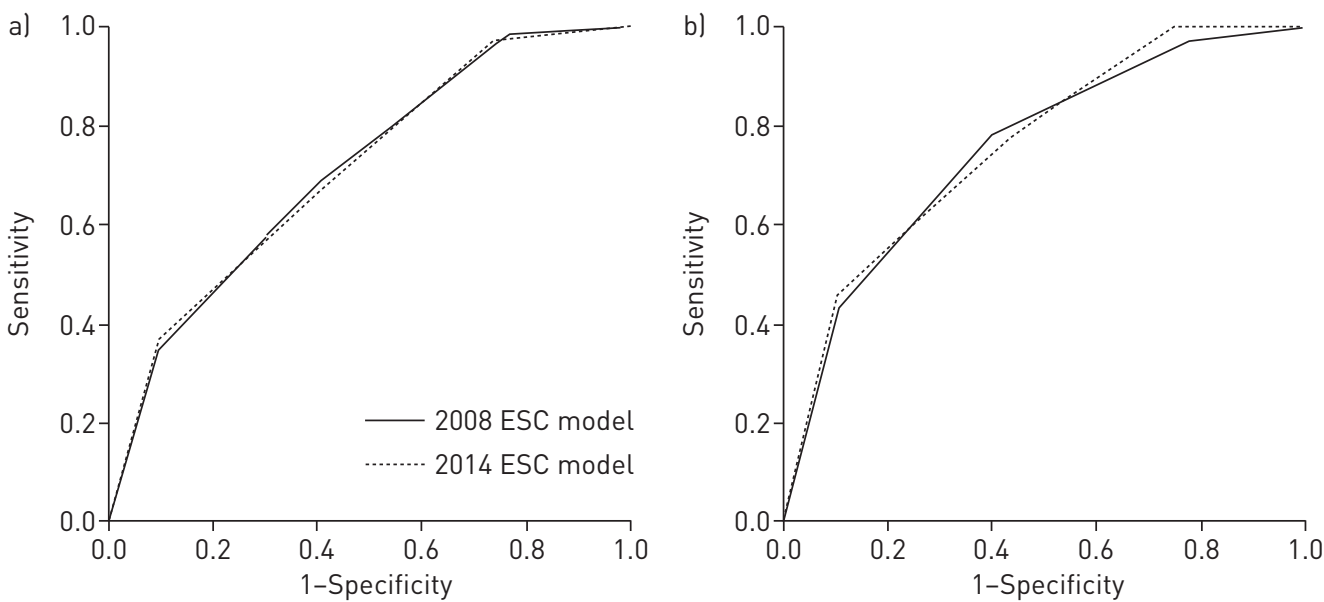

FIGURE 3 Accuracy of 2014 and 2008 European Society of Cardiology models to predict a) death at 30 days and b) death due to pulmonary embolism. 
This study has some limitations. The categorisation of patients in the four classes of risk was based on local data without a central adjudication of the RVD. Likewise, troponin was considered as normal or elevated according to local assay and cut-off values. Death was adjudicated as due to pulmonary embolism based on local evaluation. As in all observation studies, treatment was left to the discretion of the attending physicians. However, the results of the study did not change after adjusting for the use of thrombolytic therapy. Only patients with available RVD assessment and serum troponin were included in the study; thus, the number of low-risk patients (who were not supposed to proceed to further testing) might have been underestimated. We did not collect data on emerging risk stratification markers such as brain natriuretic peptide or lactate $[16,17]$. Overall, our study should be seen as a simulation of the performance of the 2014 ESC model and not as a clinical management study.

In conclusion, our study provides evidence that the 2014 ESC model efficiently stratifies patients with acute pulmonary embolism in categories at different risk of 30-day death. Management studies are required to confirm whether this integrated risk stratification model can be used to drive patient care.

\section{References}

1 Goldhaber SZ, Visani L, De Rosa M. Acute pulmonary embolism: clinical outcome in the International Cooperative Pulmonary Embolism Registry. Lancet 1999; 353: 1386-1389.

2 Heit JA, Silverstein MD, Mohr DN, et al. Predictors of survival after deep vein thrombosis and pulmonary embolism: a population-based, cohort study. Arch Intern Med 1999; 159: 445-453.

3 Konstantinides SV, Torbicki A, Agnelli G, et al., 2014 ESC Guidelines on the diagnosis and management of acute pulmonary embolism: the Task Force for the Diagnosis and Management of Acute Pulmonary Embolism of the European Society of Cardiology (ESC) endorsed by the European Respiratory Society (ERS). Eur Heart J 2014; 35 : 3033-3069.

4 Donzé J, Le Gal G, Fine MJ, et al. Prospective validation of the Pulmonary Embolism Severity Index. A clinical prognostic model for pulmonary embolism. Thromb Haemost 2008; 100: 943-948.

5 Jiménez D, Aujesky D, Moores L, et al. Simplification of the pulmonary embolism severity index for prognostication in patients with acute symptomatic pulmonary embolism. Arch Intern Med 2010; 170: 1383-1389.

6 Sanchez O, Trinquart L, Colombet I, et al. Prognostic value of right ventricular dysfunction in patients with haemodynamically stable pulmonary embolism: a systematic review. Eur Heart J 2008; 29: 1569-1577.

7 Becattini C, Agnelli G, Germini F, et al. Computed tomography to assess risk of death in acute pulmonary embolism: a meta-analysis. Eur Respir J 2014; 43: 1678-1690.

8 Becattini C, Vedovati MC, Agnelli G. Prognostic value of troponins in acute pulmonary embolism: a meta-analysis. Circulation 2007; 116: 427-433.

9 Torbicki A, Perrier A, Konstantinides S, et al. Guidelines on the diagnosis and management of acute pulmonary embolism: the Task Force for the Diagnosis and Management of Acute Pulmonary Embolism of the European Society of Cardiology (ESC). Eur Heart J 2008; 29: 2276-2315.

10 Becattini C, Agnelli G, Vedovati MC, et al. Multidetector computed tomography for acute pulmonary embolism: diagnosis and risk stratification in a single test. Eur Heart J 2011; 32: 1657-1663.

11 Becattini C, Agnelli G, Schenone A, et al. Aspirin for preventing the recurrence of venous thromboembolism. N Engl J Med 2012; 366: 1959-1967.

12 Steyerberg EW, Vickers AJ, Cook NR, et al. Assessing the performance of prediction models. A framework for traditional and novel measures. Epidemiology 2010; 21: 128-138.

13 Meyer G, Vicaut E, Danays T, et al. Fibrinolysis for patients with intermediate-risk pulmonary embolism. $N$ Engl J Med 2014; 370: 1402-1411.

14 Fernández C, Bova C, Sanchez O, et al. Validation of a model for identification of patients at intermediate to high risk for complications associated with acute symptomatic pulmonary embolism. Chest 2015; 148: 211-218.

15 Bova C, Sanchez O, Prandoni P, et al. Identification of intermediate-risk patients with acute symptomatic pulmonary embolism. Eur Respir J 2014; 44: 694-703.

16 Vanni S, Jiménez D, Nazerian P, et al. Short-term clinical outcome of normotensive patients with acute PE and high plasma lactate. Thorax 2015; 70: 333-338.

17 Lankeit M, Jiménez D, Kostrubiec M, et al. Validation of N-terminal pro-brain natriuretic peptide cut-off values for risk stratification of pulmonary embolism. Eur Respir J 2014; 43: 1669-1677. 